J. Clin. Chem. Clin. Biochem.

Vol. 20, 1982, pp. 227-234

\title{
Kritische Anmerkungen zur Auswertung von Rezeptoranalysen
}

\author{
Von E. Kuss

\section{Frauenklinik der Universität München}

(Eingegangen am 6. Februar 1982)

Zusammenfassung: Unter Berücksichtigung der wichtigsten neueren Literatur wird über die Datenverarbeitung im Rahmen der Bindungsanalysen berichtet. Aus den Grundlagen der Bindungstheorie und Informationstheorie werden für die Praxis Schlußfolgerungen zur korrekten Bestimmung von Assoziationskonstanten und Bindungsstellen-Konzentrationen abgeleitet. Die Minimierung von Fehlern wird als Funktion der Sättigung des am stärksten verdünnten Reaktionspartners betrachtet. Es wird auf die verschiedenen Möglichkeiten zur graphischen Darstellung der Bindungsfunktion hingewiesen und auf die Fehler, die sich aus mathematisch äquivalenten Umformungen der Bindungsisotherme entwickeln. Zur Parameter-Anpassung wird auf verschiedene heuristische Modelle von Bindungs-Reaktionen verwiesen und es wird empfohlen, von der praktisch generell anwendbaren Adair-Funktion auszugehen. Im Falle elektronischer Datenverarbeitung müssen gut definierte Kriterien für die Qualität der Anpassung vorgegeben sein. Die tatsächlich erreichte Qualität der Anpassung muß charakterisiert werden. Es wird auf häufig begangene, jedoch vermeidbare Fehler verwiesen.

\section{Critical comments on the calculation of receptor analyses}

Summary: The main literature concerning the processing of binding data is reviewed. Practical conclusions for the measurement of associătion constants and binding site concentrations are considered from the standpoint of basic binding theory in connection with basic information theory. The theoretical minimum errors in the association constant and the binding site concentration are discussed as a function of the saturation fraction of the most dilute component. Various plotting forms and the error arising from various rearrangements of the basic binding equation are discussed. For fitting procedure several heuristic models are mentioned and it is recommended to start from the most generàl Adair function. The necesșity of well defined criteria for quality of fit in computer programming for iterative curve fitting procedures is emphasized. The most frequent, however avoidable, errors are mentioned.

\section{Einfựurung}

Rezeptoranälysen gehören zu den Titrationsiverfähren der analytischen Chemie. Die einfachsten Beispiele werden idealisiert wie folgt beschrieben: Der eine Reaktionsteilnehmer Q (,Binder", „,Rezeptor“) mit der unbekannten fixierten Menge $\mathrm{q}$ an voneinander unabhängigen Bindungssstellen im Reaktionsvolumen wird durch Zugabe bekannter variabler Mengen $p$ an unabhängigen Bindungsstellen seines Reaktion,spartners P ( „Ligand“, „Hormon“, „Pharmakon") titriert. Kommt es zur reversiblen Assoziationsreaktion $P+Q \rightleftharpoons P Q$, so verteilt sich $q$ auf die Zustände $Q$ und $P Q$ und $p$ auf die Zustände $P$ und $P Q$. Am Ende der Reaktion haben sich die Gleichgewichtskonzentrationen $[P]$ und $[Q]$ der ,freien" und $[P Q]$ der „gebundenen“ Reaktionspartner eingestellt, deren Relation durch das Massenerhaltungsgesetz (G1. 1) und durch das Massenwirkungsgesetz (GI. 2) für bimolekulare Reaktionen definiert ist.
Die Liganden werden zumeist in leicht meßbarer Form als ,markierte “ Verbindungen, häufig als radioaktive Verbindungen mit bekannter spezifischer Aktivität $(\mathrm{Bq} / \mathrm{mol})$, eingesetzt. Deren als Meßgrößen herangezogene Konzentrationen werden ggf. in Analogie zu Gl. 1a mit $\mathrm{p}^{*}=\mathrm{P}^{*}+\mathrm{P}^{*} \mathrm{Q}$ charakterisiert, oder auch mit $\mathrm{T}^{*}=\mathrm{F}^{*}+\mathrm{B}^{*}$ (,total“, ,free“, ,bound"); $(\mathrm{p}=\mathrm{T}$, ggf. auch $\left.\mathrm{p}+\mathrm{p}^{*}=\mathrm{T}, \mathrm{P}=\mathrm{F}, \mathrm{PQ}=\mathrm{B}\right)(1,2)$.

$$
\begin{aligned}
& p=P+P Q \\
& q=Q+P Q \\
& \frac{[P Q]}{[P][Q]} \equiv K=\frac{B}{F(q-B)} \\
& \frac{B}{q}=\frac{K F}{1+K F} \equiv r ; 0 \leqslant r \leqslant 1 ; p>q \\
& r / F=-K r+K ; B / F=-K B+K q
\end{aligned}
$$




$$
\frac{B}{T}=\frac{(K T+K q+1)-\left[(K T+K q+1)^{2}-4 K^{2} q T\right]^{1 / 2}}{2 K T}
$$

Der Sättigungsgrad $r$ charakterisiert das System vollständig, wenn $p$ und $q$ bekannt sind, da $P=p$ - rq und $Q=(1-r) q$. Der Sättigungsgrad $r$ charakterisiert auch die Wahrscheinlichkeit der Bindung und mit $1-r$ die Wahrscheinlichkeit der Nicht-Bindung des Rezeptors. Die Quotienten $\mathrm{PQ} / \mathrm{p}$ bzw. 1 - $\mathrm{PQ} / \mathrm{p}$ charakterisieren diese beiden Wahrscheinlichkeiten für den Liganden; sie werden eingesetzt, wenn $\mathbf{p}<\mathbf{q}$.

Nach der Informationstheorie ergibt sich aus den beiden Wahrscheinlichkeiten für „Bindung“ und für „Nicht-Bindung " der Informationsgehalt I von $\mathrm{r}\left(\mathrm{Gl} .3 ; \mathrm{I}_{(\mathrm{r})}\right.$ in „bits“, wenn für $L$ der Wert $(\ln 2)^{-1}$ eingèsetzt wird).

$$
I_{(r)}=-L[r \ln r+(1-r) \ln (1-r)]
$$

Liegen $\mathbf{N}$ Werte von $\mathbf{r}$ vor, die sich signifikant voneinander unterscheiden, , so ist die Gesamtinformation gleich der Summe der Einzelinformationen. Die maximale Information $I_{\max }$ ist dann erreicht, wenn $\mathbf{N}$ die Menge aller sinnvollen und zugänglichen Werte von $r$ umfaßt und den gesamten Bereich von $r$ in äquidistante $r$-Werte mit Intervallen der Länge dr einteilt

$$
\begin{aligned}
I_{(N)} & =\sum_{i=1}^{i=N} I_{r_{i}}=\int_{0}^{r_{i}} I_{(r)} d r \\
I_{\max } & =\sum_{i=1}^{N} I_{r_{i}}=\int_{0}^{1} I_{r_{i}} d r
\end{aligned}
$$

Die Relation aktuelle Information/Maximalinformation akkumuliert beim Übergang von $r=0$ zu einem beliebigen Wert $\mathbf{r}=\mathbf{r}_{\mathbf{i}}$

$$
\mathrm{I} / \mathrm{I}_{\max }=\mathrm{r}-\mathrm{r}^{2} \ln \mathrm{r}+(1-\mathrm{r})^{2} \ln (1-\mathrm{r})
$$

oder, auf r normiert, (Gl. 6)

$$
I / I_{\max } r=1-r \ln r+r^{-1}(1-r)^{2} \ln (1-r) .
$$

Die Funktionen G1. 3, Gl. 5 and Gl. 6 sind in Abbildung 1 graphisch dargestellt $(3,4)$.

Für die Praxis ergeben sich aus den Gleichungen im folgenden beschriebene Konsequenzen für
I. Versuchsplanung
II. Meßwertverarbeitung
III. Auswertung

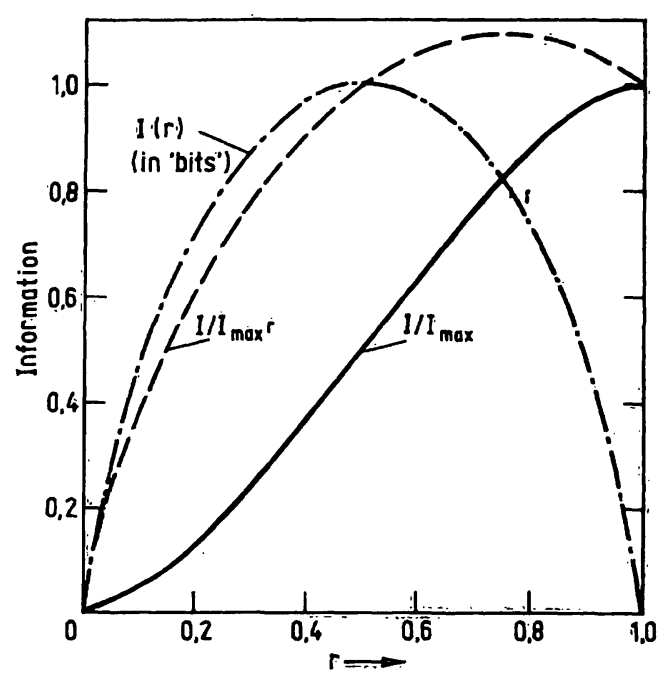

Abb. 1. Graphische Darstellung der Funktionen Gl. 3, Gl. 5 und G1. 6 .

a) Irformation $I_{(r)}$ als Funktion von $r$ (puñktiènte Kurve)

b) Akkumulierte Information relativ zur Maximalinformation $\left(\mathrm{I} / \mathrm{I}_{\max }\right.$ ) wenn $\mathrm{r}$ von $\mathrm{0}-\mathrm{i}$ ansteigt (dưxchgezogene Kurve)

c) Rate der akkumulierten Information $\left(I / I_{\max } r\right)$, wenn $r$ von $0-1$ ansteigt (gestrichelte Kurve):

(s. a. l.c. $(3,4))$

\section{Versuchsplanung}

1. Die Konzentrationen des Binders und des Liganden sollten bei etwa $1 / K$ liegen

Für die Datenverarbeitung wird angestrebt, daß der Quotient $\mathrm{B} / \mathrm{F}$ bei etwa 1 liegt; liegt er, wie nicht selten publiziert wird, bei $10^{3}$ oder $10^{-3}$, so wirken sich kleine Meßfehler sehr viel stärker aus. Nach Gl. 2 ist abzuleiten, daß B/F-Werte um 1 mit q-Werten um 1/K leicht zu erreichen sind (5). Schwierigkeiten ergeben sich bei Reạktionspartnern mit extrem hohen K-Werten u.a. deswegen, weil p* bei der üblichen spezifischen Aktivität im gesam: ten Sặttigungsbereich $\mathrm{r}$ unter der Nạchweisgrenze liegen kann. Außerdem ist zu beachten, daß bei relativ hoher Af: finität und/oder Konzentration des Rezeptors erst nach Zusatz größerer Mengen des Liganden der Bereích verlassen wird, in dem der Ligand stöchiometrisch an den Reżeptor gebunden wird (ggf. horizontaler Ast am Kurveñbeginn des Scatchard-Diagrammes $\mathrm{r} / \mathrm{F}=\mathrm{f}(\mathrm{r}) \mathrm{bzw} . \ddot{\mathrm{B}} / \mathrm{F}=$ $f(B))$ und der Bèreich erreicht wird, in dem meß̧bàre Konzentrationen an freien Liganden vorliegen. Erst in diesem Bereich ist der Formalismus für Gleichgewichtsreaktionen anwendbar. Im Bereich stöchiometrischer Addition liegt die Wahrscheinlichkeit der Bindung mit ' $P Q / p$ bei 1. Nach Gl. 3 wird die Information $I_{(r)}=0$, wenn die Wahrscheinlichkeit der Bindung 1 oder 0 beträgt.' Im Bereich der stöchiometrîischen Addition kann also keine Information gewonnen weriđ̣en (4-6). 


\section{2. r-Werte}

sollen oberhalb des Bereiches $0 \leqslant r \leqslant 0,1$ liegen

$r$-Werte im Bereich $0 \leqslant r \leqslant 0,1$ umfassen bereits $10 \%$ des Gesamtbereiches von r, ergeben aber nur $4 \%$ des Informationsmaximums. In diesem engen Sättigungsbereich kann die Relation der Meßdaten zueinander nicht als repräsentativ für den gesamten Bereich von $r$ gelten. Es besteht die Möglichkeit, in diesem Bereich für ,nearly any function, no matter how wild" fälschlicherweise eine lineare Anordnung der Meßdaten zu erkennen. Die Meßdaten dieses Bereiches dürfen aber nicht soweit ver nachlässigt werden, daß der Bindungsbereich eines zusätzlichen Binders $q^{\prime}$ mit höheren Affinitäten übersehen wird $(3,4,7)$.

\section{3. r-Werte}

sollen im Bereich $0,2 \leqslant r \leqslant 0,8$ liegen

Nach Gl. 3 liegt das Maximum für $I_{(r)}=f(r)$ bei $r=0,5$. Nach Gl. 6 wird im Bereich $r=0$ bis $r=0,76$ das Optimum der Akkumulation von Information, nämlich 83\% der Gesamtinformation, erreicht. Es ist davon auszugehen, daß etwa 75\% der Sättigungskurve definiert sein muß, bevor die Daten tatsächlich als repräsentạtiv für das untersuchte System angesehen werden können. Auch nach Gl. 5 wird offensichtlich, daß zwischen $0 \leqslant r \leqslant 0,2$ und $0,8 \leqslant r \leqslant 1,0$ die Funktion nur sehr flach ansteigt, d.h., daß in diesem Bereich der Informationszugewinn gering ist.

Der relative Fehler von $K$ wird durch $\Delta K / K=\Delta r\left[1 / r^{2}+\right.$ $\left.1 /\left(1-r^{2}\right)\right]^{1 / 2}$, der relative Fehler von $q$ wird durch $\Delta q / q=\Delta r(2 / r)^{1 / 2}$ beschrieben. Diese Funktionen zeigen, daß zwar für $q$ der Fehler bei $r=1$ minimal wird, $\mathrm{daß}$ aber in diesem Bereich der Fèhler von $K$ unendlich groß wird. Für $r<0,2$ werden die relativen Fehler von $\mathrm{K}$ und q sehr groß. Auch nạch dieser Betrachtungsweise ist zu schließen, dạ für die Bestimmung von $K$ und $\hat{q}$ der Bereich żwišchen $\mathrm{r}=0,2$ und $\mathrm{r} \equiv 0,8 \mathrm{zu}$ empfehlen ist $(3,4,7)$.

4. Die Anzahl der äquidistanten $r$-Werte soll $\hat{N}=1 / 2 s_{\dot{r}}$ betragen und möglichst hoch sein

Der in G1. 4 definierten maximalen Information kann in der Praxis nicht durch Erhöhung der Anzahl der Messun- gen beliebig nahe gekommen werden. Die Menge aller zugänglichen sinnvollen Informationen wird durch den Fehler begrenzt, mit dem $r$ bestimmt werden kann. Zwei Werte von $r$ müssen sich signifikant voneinander unterscheiden; als Signifikanzschranke wird im allgemeinen gewählt $r_{i+1}-r_{i}=2 s_{T}$, d.h. die Differenz zweier Werte von $r$ muß größer sein als die doppelte Standardabweichung von $r$. Die maximale Information ist also umso größer, je präziser die Werte von $r$ bestimmt werden können. Für das 2 Parameter-Problem (Gl. 2b), Bestimmung von $\mathrm{r}$ und $\mathrm{K}$, wird $\mathrm{N}=10$ als Minimum angegeben $(1,3,4)$.

\section{Meßwertverarbeitung}

Ziel der Meßwertverarbeitung in der Rezeptoranalyse ist, aus den Titrationskurven Informationen über die Gleichgewichtsparameter der Adsorptionsisotherme zu gewinnen. Nach Gl. 2 handelt es sich im einfachsten Fall darum, Zahlenwerte für $\mathrm{q}$ und $\mathrm{K}$ zu ermitteln. Von den zahlreichen algebraisch äquivalenten Umformungen der Hyperbelgleichung (G1. 2), die der Michaelis-MentenGleichung der Enzymologie entspricht, sind besonders die drei zu linearen Funktionen führenden Transformationen unter folgenden Namen bekannt:

Benesi-Hîldebrand: $1 / \mathrm{B}=\mathrm{f}(1 / \mathrm{F})$

(Enzymologie: Lineweaver-Burk, ,doppelt reziprok“);

Woolf-Scott: $\mathrm{F} / \mathrm{B}=\mathrm{f}(\mathrm{F})$

(Enżymologie: Hanes, ,einfach reziprok“);

Scatchard: $\mathrm{r} / \mathrm{F}=\mathrm{f}(\mathrm{r}) \mathrm{bzw} . \mathrm{B} / \mathrm{F}=\mathrm{f}(\mathrm{B})(\mathrm{Gl} .2 \mathrm{~b}),(\mathrm{Abb} .2)$ (Enzymologie: Eadie-Hofstee).

Die drei linearen Transformationen werden besonders deswegen bevorzugt angewandt, weil

1. die Ausgleichskurve, mit der die gesuchten Parameter $\mathrm{K}$ und $\mathrm{q}$ anfallen, $\mathrm{u}$. $U$. leicht zugänglich ist, sei es graphisch mit Hilfe eines Lineals oder rechnerisch mit Hilfe der linearen Regression der ,kleinsten Fehlerquadrate“,

2. die Abweichung der Ausgleichskurve von der Linearität unmittelbar anzeigt, daß die untersuchte Reaktion nicht dem durch Gl. 2 charakterisierten Modell entspricht:
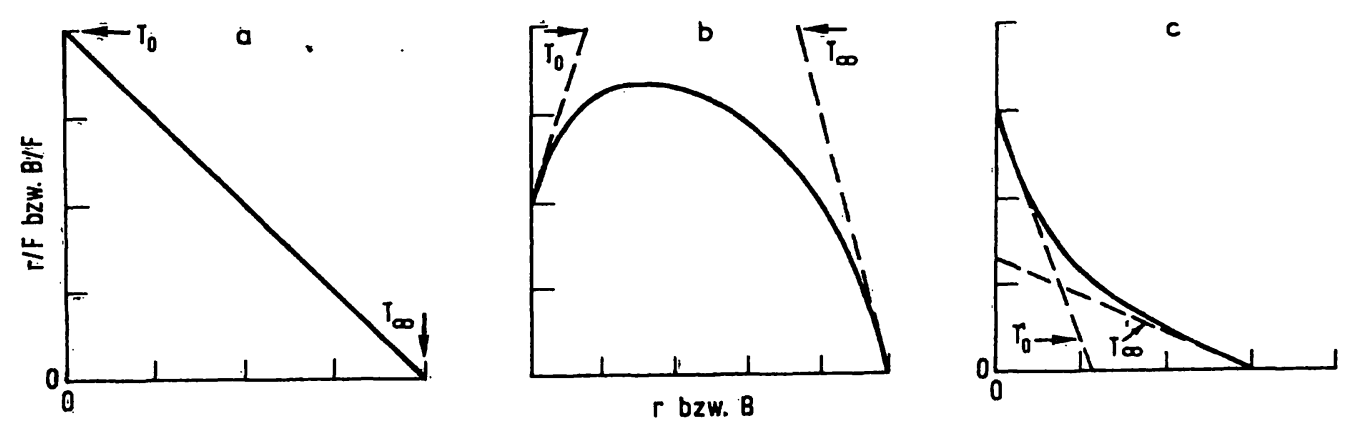

Abb. 2. Scatchard-Diagramme mit Graph und Tangenten $T_{0}$ und $T_{\infty}$.

a) linearer Graph b) konvexer Graph c) konkaver Graph (s. a. z.B. l.c. $(1,4,15-17,35)$ ). 
entweder, weil die gemessenen Konzentrationen nicht den Gleichgewichtskonzentrationen entsprachen, da entweder das Gleichgewicht noch nicht erreicht oder Fehler, z.B. bei der Trennung der Komponenten gemacht wurden (Mißklassifikation),

oder, weil die beobachtete Reaktion erheblich von dem Modell abweicht, das der Gl. 2 zugrunde gelegt wurde.

Die drei linearen Transformationen der Adsorptionsisotherme (Gl. 2) sind zwar algebraisch äquivalent, geben jedoch beim Einsetzen realer Meßdaten (z. B. für P, PQ) unterschiedliche Werte für die gesuchten Parameter (z.B. für K, q), weil die Meßfehler in Abhängigkeit von ihren Strukturen unterschiedlich stark eingehen.

Im Benesi-Hildebrand- und im Woolf-Scott-Diagramm finden die Titrationskurven keine Grenzen auf der Abszisse. Dies verleitet dazu, irgendeinen, gegebenenfalls sehr engen Ausschnitt des Sättigungsbereiches als repräsentativ darzustellen und, da Linearität angenommen wird, bedenkenlos zu extrapolieren. In der BenesiHildebrand-Transformation werden bei hohen P-Werten die Daten für $1 / \mathrm{P}$ stark komprimiert. Dies bedeutet, daß niedrige P-Werte, die mit geringer Präzision ermittelt werden, mit mehrfachem Gewicht in die lineare Regression eingehen als die präziser bestimmten höheren P-Werte. Dies bedeutet auch, daß die nur bei hoher Ligandenkonzentration meßbare sogenannte ,unspezifische Bindung", die im Diagramm nahe dem 0-Punkt plaziert ist, nicht als Abweichung von der Linearität erkannt wird, obwohl in diesen Fällen die Voraussetzungen für das der Berechnung zugrunde liegende 1:1 Modell nicht mehr erfült sind.

Im Scatchard-Diagramm (Gl. 2b, $\mathrm{r} / \mathrm{F}=\mathrm{f}(\mathrm{r})$ (Abb. 2), zumeist aber in der Form $B / F=f(B)$ ), finden die Titrationskurven ihre Grenzen an den Grenzen des ersten Quadranten. Sie beginnen und enden bei $\lim _{\mathrm{F} \rightarrow 0}(\mathrm{r} / \mathrm{F})$ und bei $\lim _{\mathbf{F} \rightarrow \infty} \mathbf{r}$.

Auf der Abszisse wird somit der gesamte Sättingungsbereich linear wiedergegeben, auch wenn $B$ aufgetragen wird und erst sekundär $q$ ermittelt und die Abszisse nachträglich auf $r$ normiert wird.

Als Nachteil der Meßwertverarbeitung nach Scatchard gilt die Tatsache, daß nicht nur die ,abhängige “ Variable $\mathrm{r} / \mathrm{F}$ bzw. $\mathrm{B} / \mathrm{F}$, sondern daß auch die ,unabhängige“ Variable $r=B / q$ bzw. B mit Meßfehlern behaftet ist und daß die Fehler der abhängigen Variablen und die der unabhängigen Variablen miteinander korrelieren (bei falsch hohem B/F wird auch falsch hohes B erhalten und umgekehrt). Daraus folgt, daß die Fehler weder in der horizontalen noch in der vertikalen Achse des Diagramms normal verteilt sind. Deshalb können auf Normalverteilung basierende statistische Berechnungen, z.B. lineare Regression nach der Methode der kleinsten Fehlerquadrate, zu falschen Ergebnissen führen $(1,4,8)$.

Im Eisenthal- und Cornish-Bowden-Diagramm können $\mathrm{K}$ und $\mathrm{q}$ über Funktionen ermittelt werden, die den obengenannten drei linearen Transformationen der Adsorptionsisotherme mathematisch äquivalent sind. Mit dieser Art der Meßwertverarbeitung kann jedoch der Vertrauensbereich der ermittelten Parameter abgeschätzt werden, ohne daß Annahmen über die Verteilung der Meßfehler gemacht werden müssen (8).'

Die mit den linearen Transformationen der Adsorptionsisotherme in Kauf genommene Fehlerhaftigkeit der $\mathrm{Ab}$ szišsenwerte kann dadurch vermieden werden (d.h. sie kann in Relation zur Fehlerhaftigkeit der Ordinatenwerte vernachlässigt werden), dạß die Adsorptionsisotherme in der Form $B=f(T)$ oder $B / T \equiv f(T)$ aufgetragen wird (Gl. 2c), da $\mathrm{T}$ mit beliebiger Präzision bestimmt werden kann $\left(B / T=\frac{B / F}{1+B / F}\right)$. Zur Berechnung der Parameter $\mathrm{K}$ und $\mathrm{q}$ liegen EDV-Programme für nichtlineare Regressionen vor. In der formal gleichartigen Analyse der Enzymkinetik kann die Konzentration dess ,freien" Substrates der Gesamtmenge an Substrat pro Volumeneinheit im allgemeinen gleichgesetzt werden, da die eingesetzte Menge an Substrat żumeist um einige Zehnerpotenzen größer ist als die eingesetzte Menge des Enzyms. In der Rezeptoranalytik darf die Konzentration des „freien" Liganden P nicht der Gesamtmenge $p$ bzw. $T=P Q+P$ des Liganden pro Ansătz gleichgesetżt wer= den, da etwa gleich große Mengen Ligand und Rezeptor eingesetzt werden. Hieraus resultiert u.a., daß beim Auftragen von $B$ als Funktion von $T$ die Halbsättigung, die beim Abszisssenwert 1/K $+q / 2$ liegt, nicht der Dissoziationskonstanten gleichgesetzt werden darf. Analoges gilt für die Charakterisienung von Kompetitoren der Bindungsreaktion. Es ist im allgemeinen nicht zulässig, die Gesamtmenge des Kompetitoris im Reaktionsansatz der Konzentration des ,freien“'Kompetitors gleichzusetzen, wie es im allgemeinen in der Enzymkinetik üblich ist, z. B. bei der Meßwertverarbeitung im Dixon-Diagiamm $(1,8)$. Da aber andererseits die Auswertung der Funktion $B=f(T)$ auf größere Schwierigkeitên stoßen kann als die Auswertung der Scatchard-Funktion, wird' vorgeschlagen, die Meßwerte zunächst im Diagramm $B=f(T)$ anzuordnen und die dadurch ermittelten Funktionswerte in das Scatchard-Diagramm $B / F=f(B)$ zur endgültigen Auswertung einzutragen $(1,9)$.

Die Meßwerte der Rezeptoranalytik werden àm häufigsten im Scatchard-Diagramm ausgewertet. Es liegen jedoch nur wenige empirische Untersuchungen über dịe Vor- und Nachteile der linearen Transformationen in der Rezeptoranalytik vor. Die divergierenden Ergebnisse sind möglicherweise Folgen unterschiedlicher Fehlerstrukturen in den Ansätzen (10-14).

\section{Auswertung von Scatchard-Funktionen}

Ein gegebener Satz von Meß̣daten kann näherungsweise durçh zahlreiche Funktionen beschrieben werden, die entweder von definierten physikalisch-chemischen Reaktionsmodellen abgeleitet wurden oder rein mathema- 
tisch-statistische Regressions- oder Interpolationsmethoden darstellen (Spline-Funktionen, Polynome). Gewisse Funktionen lassen sich nicht explicit, sondern nur durch iterative Rechenverfahren mit Hilfe der EDV lösen. Selbst optimale Ubereinstimmung von Funktion und Meßdaten beweist nicht zwingend die Ubereinstimmung der den Funktionen zugrundeliegenden Modellvorstellung mit dem untersuchten realen System.

Zur Auswertung der Meßdaten einer Rezeptor-Analyse werden zunächst die Tangenten $T_{0}$ und $T_{\infty}$ des ScatchardGraphen bei $\lim _{F \rightarrow 0}(r / F)$ bzw. $\lim _{F \rightarrow \infty}(r)$ konstruiert, ggf.

berechnet. Entweder die Tangenten fallen mit dem Scatchard-Graphen zusammen (linearer Scatchard-Graph) oder sie fallen nicht zusammen (nicht-linearer ScatchardGraph) (Abb. 2).

\section{Auswertung linearer Scatchard-Graphen}

Wenn die Tangenten $T_{0}$ und $T_{\infty}$ mit dem ScatchardGraphen zusammenfallen, ist die notwendige Bedingung dafür erfüllt, daß die untersuchte Reaktion dem

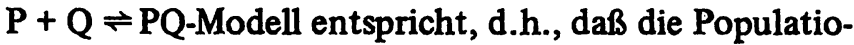
nen der Reaktionspartner $\mathrm{p}$ und $\mathrm{q}$ hinsichtlich der Affinitäten zueinander aus homogenen, unabhängigen Bindungsstellen bestehen. Dann ist nach $\mathrm{Gl} .2 \mathrm{~b}$, wie vielfach beschrieben,

der Anstieg von $T_{0}=$ Anstieg von $T_{\infty}=$ Anstieg des Scatchard-Graphen $=-\mathbf{K}$,

der Abszissenabschnitt von $T_{0}=$ Abszissenabschnitt von $T_{\infty}=$ Abszissenabschnitt des Scatchard-Graphen $\mathrm{r}=1$ bzw. $B=q$ und

der Ordinatenabschnitt $\vec{T}_{0}=$ Ordinatenabschnitt $T_{\phi \infty}=$ Ordinatenabschnitt des Scatchard-Graphen $=\mathrm{K}$.

Linearitätt des Scatchard-Graphen kann jedoch u.U. auch auftreten, wenn in einer heterogenen Rezeptor-Population die Liganden-Affinität breit um eine mittlerèe Affinität gestreut ist. Der Ordinaten-Abschnitt entspricht dann der mittleren Assożiationskonstante $\overline{\mathrm{K}}$ (18).

\section{Auswertung nicht-linearer $S c a t c h a r d-G r a p h e n$}

\section{A) Mißklassifikationen}

Bevor andere Ürsachen der Nicht $=\dot{L}$ inearität des Scatchard-Graphen diskutiert werden, muß sichergestellt sein, daß die Meßw werte tatsächlich Gleichgewichtskonzentrationen repräsentieren. Abweịchungen von diesen Voraussetzungen kommen relativ häufig vor, $z$. B. auch als Ursache des ,hook-Effektes", der mit positiver Kooperation verwechselt werden kann. B wird nach Verlusten (z.B. Adsorption, Zersetzung von Binder oder Komplex) zu niedrig oder nach Verunreinigung mit $F$ (z.B. Co-Präzipitation) zu hoch bestimmt. Häufig wird der unbesetzte Rezeptor schneller zersetzt als seine komplexierte Form. Die zur Einstellung des ReaktionsGleichgewichtes ermittelte Zeit muß nicht notwendig auch für Ansätze mit anderer Konzentration der Reaktionspartner guiltig sein $(12,14,19-21)$.

\section{B) Abweichung vom Modell $P+Q \rightleftharpoons P Q$}

Wegen der Äquivalenz von $\mathrm{P}$ und $\mathrm{Q}$ wirken sich Abweichungen von der Univalenz, Homogenität, Unabhängigkeit der Bindungsstellen von Ligand und Rezeptor gleichartig aus. Die prinzipielle Austauschbarkeit beider GröBen wird nur dadurch eingeschränkt, daß z.B. der markierte Ligand als Meßgröße dient und nicht der Rezeptor. Der Ligand wird als Reagenz ausgewählt, es kann jedoch nicht immer davon ausgegangen werden, daß der zur Rezeptoranalytik eingesetzte Ligand ebenso gut charakterisiert ist wie andere ,p.A.-Reagenzien“.

Systeme, deren Heterogenität mit n Klassen unabhängiger Bindungsstellen des Liganden,

$$
p=\sum_{i=1}^{n} p_{i} \quad(i=1,2, \ldots, n)
$$

und mit $\mathrm{m}$ Klassen unabhängiger Bindungsstellen des Rezeptors,

$$
q=\sum_{j=1}^{m} q_{j} \quad(j=1,2, \ldots, m),
$$

definiert werden kann, werden durch einen Satz von Gleichungen (Gl. $2 b^{\prime}$ ) beschrieben, in denen Gl. $2 b$ als Spezialfall $(n=1, m=1)$ enthalten ist $(22)$.

$$
\frac{B_{i}}{F_{i}}=\sum_{j=1}^{m} \frac{K_{i j} q_{j}}{1+\sum_{a=1}^{n} K_{a j} F_{a}}
$$

\section{a) Heterogenität des Liganden}

Absichtlich wird die Heterogenität der Liganden in den „Kompetitionsverfahren" der Rezeptoranalytik eingesetzt, wenn der zur leichteren Meßbarkeit besonders „markierte“ Ligand p* sich im Reaktionsansatz aufgrund seiner Markierung oder seiner geringen Menge anders verhält als nur als Ligand des zu analysierenden Rezeptors $q(1,6,14,23)$. Es wird dann versucht, diese „unsspezifischen “ Effekte dadurch zu eliminieren, daß die „spezifische“ Bindung des markierten Liganden durch Zusatz steigender Mengen des unmarkierten $\mathrm{Li}$ ganden gehemmt wird, bis nur die „unspezifische“ Bindung übrig bleibt. In dieser Form der Bindungsanalysen wird jetzt $p_{\text {tot }}=p^{*}+p$ und $q=Q+P * Q+P Q$ (bei konstantem $p^{*}$ variiert die spezifische Aktivität der variablen Dosis p). Wenn markierter und unmarkierter Ligand gleiche Affinität zum Rezeptor aufweisen $\left(\mathrm{K}^{*}=\mathrm{K}\right)$, resultiert daraus ein linearer Scatchard-Graph mit dem Anstieg $-K^{*}=-K$ und dem Abszissenabschnitt $q$, unabhängig davon, wie groß $\mathrm{p}^{*}$ gewählt wurde. Wenn die Affinitäten von markiertem und unmarkiertem Ligand differieren, resultiert daraus bei $K^{*}>K$ ein nach unten, bei $\mathrm{K}^{*}<\mathrm{K}$ ein nach oben durchgebogener Scatchard- 
Graph, dessen Anstieg und Abszissenabschnitt u.a. von der eingesetzten Menge $\mathrm{p}^{*}$ abhängt. Zum Ausschluß der Hypothese $\mathrm{K}^{*}$ \# $\mathrm{K}$ werden Rezeptoranalysen als Kompetitionsverfahren angesetzt, und zwar in mehreren Serien mit unterschiedlichen Mengen von p* (z.B. 1/10 p*, $\left.p^{*}, 10 p^{*}\right)$. Kompetitionsverfahren werden auch dann eingesetzt, wenn die spezifische Aktivität von $\mathrm{p}^{*}$ ermittelt werden soll. Dies und die Prüfung der Hypothese $\mathrm{K}$ \# $\mathrm{K}^{*}$ ist besonders dann zu empfehlen, wenn Liganden eingesetzt werden, die mit Heteroatomen, wie z.B. ${ }^{125}$ I markiert sind (24-28).

Heterogenität des Liganden kann auch unbeabsichtigt auftreten, sei es durch die Markierung, z.B. können bei 4 Tyrosin/Molekül (Insulin) theoretisch 80 I-Derivate entstehen, oder sei es durch Selbst-Aggregation bei höheren Konzentrationen von p (29). Es ist ebenfalls zu beachten, daß der Ligand auch erst während der Inkubation als Folge von Nebenreaktionen heterogen geworden sein kann.

\section{b) Heterogenität des Rezeptors}

(Heterogenität präexistent oder durch Bindung induziert)

Wenn per exclusionem die Nicht-Linearität des ScatchardGraphen auf Heterogenität der Bindungsstellen des Rezeptors zurückgeführt worden ist, wird die weitere Präzisierung eines geeigneten Modells davon abhängig gemacht, ob der Graph ein Maximum besitzt oder nicht $(1,2,3,15-17,29)$.

Ein nach oben gebogener konvexer Scatchard-Graph (,concave down") mit einem Maximum (negative 2. Ableitung) kann bekanntlich als Folge positiver Kooperativität der Bindungsstellen entstanden sein. Aus der Lage des Maximums kann auf das Ausmaß der positiven Kooperativität geschlossen werden; für weitergehende Aussagen wird empfohlen, die Meßwerte in ein SipsHill-Diagramm einzusetzen $(17,29)$.

Ein nach unten gebogener konkaver Scatchard-Graph (,concave upward“) kann als Folge negativer Kooperativität und/oder als Folge praeexistenter Heterogenität mit diskreter oder kontinuierlicher Verteilung der Bindungsenergien entstanden sein. Weitergehende Schlüsse über den molekularen Mechanismus der Bindungsreaktion können aus dem Verlauf des Scatchard-Graphen allein nicht gefolgert werden.

Systeme mit praeexistenter und/oder induzierter Heterogenität der Rezeptor-Bindungsstellen werden im allgemeinen durch die Adair-Funktion (Gl. 2a') beschrieben, die Gl. $2 a$ als Spezialfall $(j=1, \psi=K)$ enthält $(1-4,15-17,29)$.

$$
\mathrm{r}=\frac{\sum_{\mathrm{j}=1}^{\mathrm{m}} \mathrm{j} \psi_{\mathrm{j}} \mathrm{F}^{\mathrm{j}}}{1+\sum_{\mathrm{j}=1}^{\mathrm{m}} \psi_{\mathrm{j}} \mathrm{F}^{\mathrm{j}}}
$$

Aus Gi. $2 a^{\prime}$ wird $\frac{d(r / F)}{d r}$ abgeleitet, und damit werden auch die Parameter der Tangenten $T_{0}$ und $T_{\infty}$ im $S$ catchard-Diagramm gewonnen (Abb. 2).

Die numerischen Werte für die Adair-Konstanten $\psi_{\mathbf{j}}$, für die phänomenologischen Konstanten zut Beschreibung der Bindung des $\mathrm{j}^{\text {ten }}$ Liganden, können aus den Anstiegen, Ordinaten- und Abszisșenabschnitten der Tangènten $T_{0}$ und $T_{\infty}$ gewonnen werden. Inre Interpretation als Terme, zusammengèsetzt aus makroskopischen (molekularen, stöchiometrischen, Titrations-) und mikroskopischen (Bindungsstellen-) Assoziationskonstanten, aus statistischen Faktoren, aus Koeffizienten der Variation und İndices der Heterogenität zur Beschreibung dèr Dispersion eines Affinitäts-Spektrums u. a., ist vọm unterlegten Modell abhängig. Die korrekte Konstruktion der Tangenten und der richtige Zusammenhang zwischen den Parametern der Tangenten und der möglichen reaktionsmechanistischen Deutung der Adair-Konstanten ist mehrfach beschrieben worden $(1-5,14-17,29)$; trotzdem kommt es, selbst in Publikationen renommierter Zeitschriften, häufig zu falschen Konstruktionen und Interpretationen (30), Besonders oft wird der Anstieg von $T_{0}$ fälschlich als Wert der Assoziationskonstanten $\left(-K_{1}\right)$ und der zugehörige Abszissenabschnitt fälschlich als Wert der Menge $q_{1}$ der Bindungsgruppe mit der stärksten Affinität bezeichnet sowie der Anstieg von $T_{\infty}$ fälschlich als $=K_{m}$ der Bindungsgruppe $q_{m}$ mit der schwächsten Affinität bezeichnet.

Ein ebenfalls als ,unspezifische Bindung“( NSB $_{\text {graph }}$ ) bezeichneter Anteil an den Meßwerten für B kann graphisch über $\lim _{B \rightarrow \infty} r / F=N B_{\text {graph }} F$ eliminiert werden $(14,31,32)$. Diese Annahme einer Asymptote parallèl zur $x$-Achse läßt sich nach Gl. 2b' ableiten:

$$
\begin{aligned}
& \mathrm{n}=1 ; \mathrm{m} ; \\
& \frac{\mathrm{B}}{\mathrm{F}}=\frac{\dot{\mathrm{K}}_{1} \mathrm{q}_{1}}{1+\mathrm{K}_{1} \mathrm{~F}}+\ldots+\frac{\mathrm{K}_{\mathrm{m}} \mathrm{q}_{\mathrm{m}}}{1+\dot{\mathrm{K}}_{\mathrm{m}} \mathrm{F}} ; \\
& \mathrm{K}_{\mathrm{m}} \ll \mathrm{q}_{\mathrm{m}}: \frac{\mathrm{K}_{\mathrm{m}} \mathrm{q}_{\mathrm{m}}}{1+\dot{\mathrm{K}}_{\mathrm{m}} \mathrm{F}}=\mathrm{K}_{\mathrm{m}} \mathrm{q}_{\mathrm{m}}=\text { const. }
\end{aligned}
$$

Es empfiehlt sich āber, die ,unspezifịsche Bindung “ auch experimentell zu bestimmen, d.h. Bindungskurven von $\mathrm{p}^{*}$ mit und ohne Zusatz von überschüssigem unmarkierten Liganden $p$ żu erstellen und die so ermittelten Differenzen als. Funktion von $p^{*}$ aufzutragen. Wenn eine lineare Beziehung nachzuweisen ist, sind die Funktionswerte der unspezifischen Bindung ( $\mathrm{NSB}_{\text {exp }}$ ) besser als Korrekturglied der ,korrigierten" Scatchard-Funktion

$$
\text { - } \frac{B_{\text {tot }}-N S B_{\text {exp }}}{T-B_{\text {tot }}}=f\left(B_{\text {tot }}-N S B_{\text {exp }}\right)
$$


Wolfgang Voelter Günter Weitzel (Editors)

\section{Structure and Activity of Natural Peptides Selected Topics}

Proceedings of the Fall Meeting

Gesellschaft für Biölogische Chemie

Tübingen, Germany, September 1:979

$1981.17 \mathrm{~cm} \times 24 \mathrm{~cm}$. XII, 648 pages. Numerous illustratioñs.

Hardcover. DM 150,-; approx. US $\$ 83.50$

ISBN 3110082640

This volume is the result of selected contributions presented at the Fall Meeting of the Gesellschaft für Biologische Chemie in Tübingen in 1979.

CONTENTS (main chapters):

I. Surveys of Selected Topics. II. Isolation of New Peptides.

III. Methods of Purification, Isolation and Characterization of Peptides.

IV. Peptide Syntheses. V. Miscellaneous and Biological Activity of Pep̃tides
Das Kreiskrankenhaus Bergstraße

in Heppenheim

- Inbetriebnahme 1. Juli 1982 -

sucht

zum nächstmöglichen Einstellungstermin

1 Chemiker (Laborleiter)/

1 Leitende med. techn. Assistentin (Labor)

Das Kreiskrankenhaus

verfügt über 373 Planbetten für die Bereiche

Innere Medizin I und II

Chirurgie

Orthopädie

Gynäkologie

Bewerbungen mit den üblichen Unterlagen sind $z u$ richten an das

Kreiskrankenhaus Bergstraße

Viernheimer Straße 2

6148 Heppenheim

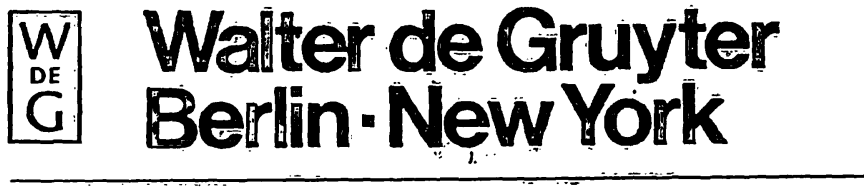

E. Buddecke

\section{Biochemische Grundlagen der Zahnmedizin}

$17 \mathrm{~cm} \times 24 \mathrm{~cm} . X V, 193$ Seiten. 90 Abbildungen. 19 Täbellen. 1981. Flexibler Einband. DM 36,ISBN 3110087383

Das Kurzlehrbuch für Zahnärzte und Studierende behandelt die Biochemie der Zähne, des . Zahnhalteapparates und der Mundhöhle. Es. werden Chemie und Stoffwechsel der organišchen Mațix der Zahnhartsubstanz, Biomineralisàtion, Fluoridstoffwechsel und die spezzielle Biochemie des Speichels und der Mikrooorganismen der Mundhöhle beschrieben. Sie bilden die Grundlage für die Pathobiochèmie der beiden häufigsten

Erkrankungen der Odontologie - der Karies und dér Parodontopathie. Die Darstellung umfaßt neben pathogenetischen auch präventive Aspekte der Karies und Parodontopathie sowie eine Ubersicht über die chemische Zusammensetzung und Wirkungsweise von Zahnpflegemitteln.

Der behandelte Inhalt des. B̉uches berücksichtigt auch die Prüfungsordnung für Zahnärzte. 
geeignet als die Einzelwerte oder gemittelten Einzelwerte der unspezifischen Bindung. Aber auch diese Methode der experimentellen Ermittlung von NSB ist nicht unproblematisch, insbesondere, wenn die als $p$ eingesetzte Substanz sich von p $^{*}$ nicht nur durch die Radioaktivität unterscheidet. Andere Verfahren zur experimentellen Bestimmung von NSB wie z.B. die über Hitze-Inaktivierung der Rezeptoren sind ebenfalls nicht unproblematisch. Auf die alternative Möglichkeit, die spezifische Rezeptorbindung $B_{\text {spez }}$ dadurch zu bestimmen, daß der Effekt $\mathrm{E}$ spezifischer Folgereaktionen gemessen wird $E=f\left(B_{\text {spez }}\right)$, sei ebenfalls hingewiesen $(14,23,33-35)$.

Die prinzipiellen Schwächen der graphischen Methoden zur Bestimmung der Parameter der Liganden-Bindung durch Rezeptoren liegen darin begründet, daß, von der „unspezifischen“ Bindung abgesehen, nicht mehr als vier Parameter eines Systems bestimmt werden können (z.B. $K_{i 1}, K_{i m} ; q_{1}, q_{m}$ ) und daß zur Konstruktion der Tangenten $T_{0}$ und $T_{\infty}$ ausgerechnet die Regionen des Scatchard-Graphen herangezogen werden muissen, die nur mit großer Unsicherheit bestimmt werden können ( $<<0,1$ bzw. $>0,9)$. Schließlich läßt sich Subjektivität beim Konstruieren der Tangenten nicht ausschließen und die Definition eines Vertrauensbereiches der Parameter kaum verwirklichen.

Mit Hilfe der modernen Möglichkeiten der Datenverarbeitung können die Parameter beliebiger mathematischstatistischer Funktionen so variiert werden, daß sie sich sämtlichen Meßpunkten der Rezeptoranalyse optimal anpassen. Damit besteht die Möglichkeit, den gesamten Meßbereich zu nutzen, um für Systeme beliebiger Komplexizität Lösungen zu finden (Abb. 3). Wenñ aber, wie bereits erwähnt, mindeștens 10 Meßpunkte erforderlich sind, um eine zuverlässige Anpassung einer Geradengleichung mit zwei unbekannten Parametem $(K, q)$ zu gewährleisten, und wenn andererseits die Anżāhl sinnvooller Meßpunkte $N$ durch $N_{\max }=1 / 2 \mathrm{~s}$, begrenzt ist, wird es offensichtlich, daß auch mit Hilfe der elektronischen
Datenverarbeitung selten Zahlenwerte für mehr als 5 Parameter ermittelt werden können, für die über 25 Meßpunkte gefordert werden (1). Es muß betont werden, daß auch die elektronische Datenverarbeitung die Information nicht produziert, sondern aus den Meßwerten extrahiert. Die Subjektivität oder Willkür kann auch mit elektronischer Datenverarbeitung Eingang in die Rezeptoranalytik finden, wenn nicht die optimale Parameter-Anpassung an die Meßdaten adäquat definiert und getestet wurde (35-37). In Abb. 3 sind neben Beispielen idealer Parameteranpassung auch die wichtigsten Abweichungen vom idealen ,fit" dargestellt. Die entsprechenden Diagnosen wurden dadurch der Willkür entzogen, daß reale Meßdaten geeigneten Prüfprogrammen unterworfen wurden (36).

Zur Interpretation der vom Rechner aufbereiteten Information ist es notwendig, sich u.a. mit den Theorien der Reaktionskinetik, wie auch mit den Theorien der optimalen Parameter-Anpassung auseinander zu setzen, um z.B. die Plausibilität des Reaktionsmodells abzuwägen, und um zu prüfen, wie weit z. B. Normalverteilung und Homoscedastizität der Meßfehler vorausgesetzt wird $(1,4,7$, $22,31,35-37)$. Die vorherige kritische graphische Auswertung der Meßwerte am eigenen Laborplatz fördert nicht nur die unmittelbare Einsicht in die Problematik der Rezeptoranalytik, sie ist auch hilfreich, um die initialen Parameter für die Iterationsverfahren des Rechners bereitzustellen. Auch wenn die Möglichkeit gegeben ist, mit Hilfe vorgegebener Programme die Daten der Rezeptoranalysen außerhalb des Laboratoriums auszuwerten bzw. auswerten zu lassen, sollte das Labor also über ein Basiswissen hinsichtlich der mathematischen Grundzüge der Assoziationsreaktionen verfügen, dessen Umfeld hier abgesteckt werden sollte. Das Lesen dieses Beitrags kann und soll das Studium der (ausnahmsweise und beabsichtigt mit Titel) aufgeführten ('bersichten und Originalarbeiten nicht ersetzen, es kann und soll aber dazu beitragen, Probleme der Praxis zu erkennen und so die jetzt

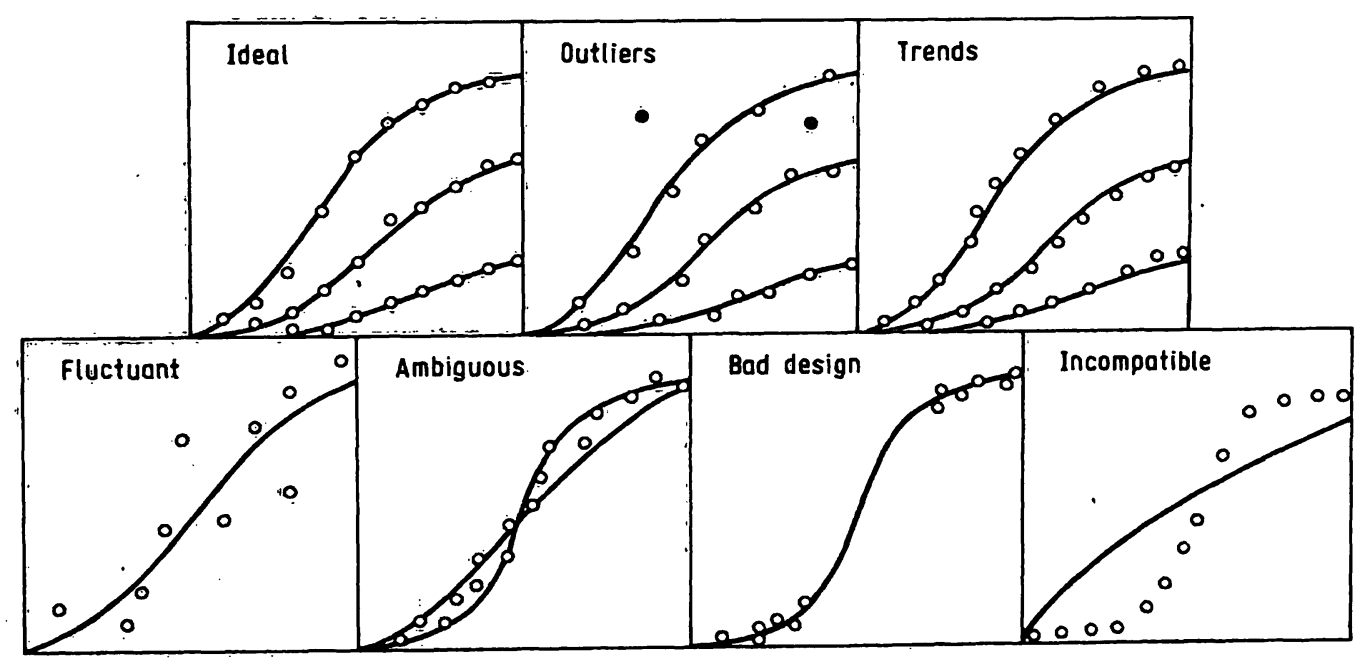

Abb. 3. Tests auf Passung und Beispiele unbefriedigender Passung zwischen den Parametern von theoretischem Modell und realem System (aus 1.c. (36)). 
noch so zahlreich publizierten Verstöße gegen mathematisch-statistische Regeln in der Rezeptoranalytik zu vermindern (30). Der Ausspruch „There is nothing more practical than a good theory“ (Leonid Breshnew, zit. nach 1.c. (38) kann auch auf die Laborpraxis der Rezeptoranalytik bezogen werden.

\section{Literatur}

1. Rodbard, D. (1973) Mathematics of hormone-receptor interaction: I. Basic principles.

Rodbard, D. \& Bertino, R. E. (1973) Theory of radioimmunoassays and hormone-receptor interactions: II. Simulation of antibody divalency, cooperativity and allosteric effects. In: Receptors for reproductive hormones (O'Malley, B. W. \& Means, A. R., eds.) New York: Plenum Press, pp. 289341. (Advanc. Exp. Med. Biol., Vol. 36).

2. Winkler-Oswatitsch, R. \& Eigen, M. (1979) Die Kunst zu Titrieren. Vom klassischen Endpunktverfahren zur modernen differentiellen und dynamischen Analyse. Angew. Chem. 91, $20-51$.

3. Weber, G. \& Anderson, S. R. (1965) Multiplicity of binding. Range of validity and practical test of Adair's Equation. Biochemistry 4, 1942-1947.

4. Deranleau, D. A. (1969) Theory of the measurement of weak molecular complexes: I. General considerations. II. Consequences of multiple equilibria. J. Amer. Chem. Soc. 91, 4044-4054.

5. Buller, R. E., Schrader, W. T. \& O'Malley, B. W. (1976) Steroids and the practical aspects of performing binding studies. J. Steroid Biochem. 7, 321-326.

6. Cuatrecasas, P. \& Hollenberg, M. D. (1976) Membrane receptors and hormone action. In: Advances in protein chemistry (Anfinsen, C. B., Edsall, J. T. \& Richards, F. M., eds.) Vol. 30. New York: Academic Press, pp 251-428.

7. Weder, H. G., Schildknecht, J., Lutz, R. A. \& Kesselring, P. (1974) Determination of binding parameters from Scatchard plots. Theoretical and practical considerations. Eur. J. Biochem. 42, 475-481.

8. Atkins, G. L. \& Nimmo, I. A. (1980) Current trends in the estimation of Michaelis-Menten Parameters. Anal. Biochem. 104, 1-9.

9. Thakur, A. K. \& Rodbard, D. (1979) Graphical aids to interpretation of Scatchard plots and dose-response curves. J. Theor. Biol. 80, 383-403.

10. Cressie, N. A. C. \& Keightley, D. D. (1979) The underlying structure of the direct linear plot with application to the analysis of hormone-receptor interactions. J. Steroid Biochem. 11, 1173-1180.

11. Keightley, D. D. \& Cressie, N. A. C. (1980) The Woolf plot is more reliable than the Scatchard plot in analysing data from hormone receptor assays. J. Steroid Biochem. 13, 1317-1323.

12. Nimmo, I. A., Atkins, G. L., Strange, R. C. \& Percy-Robb, I. W. (1977) An evaluation of ways of using equilibrium dialysis to quantify the binding of ligand to macromolecule. Biochem. J. 165, 107-110.

13. Woosley, J. T. \& Muldoon, T. G. (1977) Comparison of the accuracy of the Scatchard, Lineweaver-Burk and direkt linear plots for the analysis of steroid-protein interactions. J. Steroid Biochem. 8, 625-629.

14. Biaunsberg, H. \& Hammond, K. D. (1980) Practical and theoretical aspects in the analysis of steroid receptors. J. Steroid Biochem. 13, 1133-1145.

15. Klotz, I. M. \& Hunston, D. L. (1971) Properties of graphical representations of multiple classes of binding sites. Biochemistry 10, 3065-3069.

16. Henis, Y. I. \& Levitzki, A. (1976) An analysis on the slope of Scatchard plots. Eur. J. Biochem. 71, 529-532.

17. Dahlquist, F. W. (1978) The meaning of Scatchard and Hill plots. Meth. Enzymol. 48, Enzyme structure, pt. F, pp270299.

18. Minton, A. P. (1979) On the interpretation of binding isotherms in complex biological systems. Apparent homogeneity of some heterogenous systems. Biochim. Biophys. Acta
$558,179-186$.

19. Swillens, St. \& Dumont, J. E. (1975) A pitfall in the interpretation of datá on ligañd-protein interaction. Biochẹm. J. 149, 779-782.

20. Saiduddin, S. \& Zạssenhaus, H. P. (1978) Further consideriation of systematic errors present in protein-ligand interactions. Steroids 32, 51-59.

21. Borth, R. (1980) Masss-action model for radioimmununoassays and other saturation assays with atypical performance characteristics. Math. Biôsci. 51, 187-197.

22. Feldman, H. A. (1972) Mathematical theory of complex ligand-binding systems at equilibrium: Some methods for parameter fitting. Anal. Biochem. 48, 317-338.

23. Cuatrecasas, P. \& Hollenbeṙ̆, M. D. (1975) Binding of insulin and other hormones to non-receptor materiăls: Sătứability, specificity and apparent "negative cooperativity". Biochem. Biöphys. Res. Commun. 62, 31-41.

24. Akera, T. \& Cheng, V..J. K. (1977) A simple method for the determination of affinity and binding site concentration in receptor binding studies. Biochim. Bî̉ophỳs. Actậ 470, 412423.

25. Walker, W. H. C. (1977) An approach to immunóasșay. Clin. Chem. $2 \overline{3}, 384-402$.

26. Taylor, S. I. (1975) Binding of hormones to receptọis. An alternative explanation of nonlinear Scatchard plots. Biochemistry $14,2357-2361$.

27. Hollemans, H. J. G. \& Bertina, R. M. (1975) Scatchard plot and heterogeneity in binding affinity of labeled and un= labeled ligand. Clin. Chem. 21, 1769-1773.

28. Verhoff, F. H., Lisi, P. J., Fischer, C. D., Teipel, J. W., Goldstein, G. \& Schiffman, M. B. (1980) Graphical detërmiña= tion of specific activity, binding connstants, and antibodysite concentrations for radioimmunoassays, with application to thymopoietin. Clin. Chem. 26,718-723.

29. Thakur, A. K., Jaffe, M. L. \& Rodbard; D. (1980) Graphical analysis of ligand-binding systems: Évaluation by Mönte Carlo Studies. Anal. Biochem. 107;279=295.

30. N $\phi$ rby, J. G., Ottolenghi, P. \& Jensen, J. (1980) Scatchàrd plot: Common misinterpretation of binding experimentents. Anal. Biochem. 102, 318-320.

31. Schwarz, S. (1979) A simple computer program for Scatchard plot analysis of steroid receptors including non-specific binding correction on a low cost desk top calculator. J. Steroid Biochem. 11, 1641-1646.

32. Braunsberg, H. \& Hammond, K. D. (1979) Methods of steroid receptor calculation: An interlaboratọtry study. J. Steroid Biochem. 11, 1561-1565.

33. Strickland, S. \& Loeb, J. N. (1981) Obligatory separation of hormone binding and biological response curves in systems dependent upon secondary mediators of hormone action. Proc. Natl. Acad. Sci. U.S.A. 78, 1366-1370.

34. Tallarida, R. J. (1981) Theoretical pharmacology: the quantitative identification of receptors. Trends Pharmacol. Sci. (TIPS) 2, 231-234.

35. Rodbard, D., Munsoñ, P. J. \& Thakur, A. K. (1980) Quantitative characterization of hormone receptors. Canceì 46 , 2907-2918.

36. Reich, J. G., Wangermann, G., Falck, M. \& Rohde, K. (1972) A general strategy for parameter estimation from isosteric and allosteric-kinetic data and binding measure: ments. Eur. J. Biochem. 26, 368-379.

37. Atkins, G. L. (1976) Tests for the goodness of fit of models, Biochem. Soc. Transaction 4, 357-361.

38. Rich, V. (1977) Following a Maoist path. Nature 270, 470471 .

Prof. Dr. Dr. Erich Kuss I. Frauenklinik dẹ Universität Maistr., 141 D-8000 München 2 